\title{
La constitución de una memoria terminológica: elaboración de terminologías bilingües en programas de memoria de traducción
}

\author{
Carles Tebé \& María Teresa Cabré \\ Universitat Pompeu Fabra, Barcelona
}

Computer-aided translation systems (CAT) based on Translation Memories (TM) are a widely diffused technology that uses database and code-protection features to improve the quality, efficiency and consistency of the human translation process. These systems basically consist of a textual database in which each source sentence of a translation is stored together with the target sentence (this is called a translation memory "unit"). New and changed translation proposals will then be stored in the database for future use. This textual database - the kernel of the system - is combined with a terminological database (TDB), which is used by translators to store independently, terminological equivalences or translation units of particular value.

In this paper the authors outline a first draft of a methodology that describes the preparation of a bilingual terminology from - and within - TM applications. The bilingual corpus produced is called the 'terminological memory' of the translator.

\section{Introduction}

En esta comunicación los autores presentamos una propuesta metodológica de elaboración de terminologías bilingües a partir de la traducción de textos especializados en sistemas de traducción asistida basados en memorias de traducción. La terminología resultante de este proceso la denominamos memoria terminológica del traductor.

Los sistemas de traducción asistida por ordenador (TAO) basados en memorias de traducción (SMT) han experimentado los últimos años un auge muy importante, y se implantan con gran velocidad en la mayoría de entornos profesionales que resuelven traducciones especializadas. Las aplicaciones de SMT constan de una estructura modular que integra, entre otros elementos, un entorno de traducción bilingüe y un gestor de bases de datos terminológicos. Hasta el momento, sin embargo, no hay trabajos publicados ni modelos pedagógicos que expliquen cómo interactúan textos y unidades terminológicas en estos nuevos entornos de trabajo, ni cómo deben retroalimentarse mutuamente.

El modelo de interacción que presentamos y que hemos desarrollado en nuestros trabajos consiste en desarrollar una metodología de trabajo que integre en un mismo proceso la elaboración de traducciones y de 
glosarios terminológicos para la traducción. A partir de las propuestas de Tebé, Cabré y Quiroz (2002), que presentan las posibilidades de gestión integrada de textos y términos que permiten las nuevas herramientas específicas para la traducción especializada, y Tebé (2003), que analiza sus posibilidades en la didáctica de la traducción especializada, los autores exponemos cómo se elabora una terminología bilingüe a partir de un corpus bilingüe alineado desde una memoria de traducción.

Concretamente, nos proponemos presentar qué recursos ofrecen las MT para gestionar la terminología junto a la traducción especializada, qué debe modificarse de la metodología de trabajo en terminología para integrar la terminología en la traducción asistida por ordenador, cuáles son algunas de las consecuencias que tendrá la aplicación de esta nueva metodología en el ámbito de la traducción especializada.

\section{Los glosarios terminológicos en la traducción}

En un trabajo preliminar sobre este tema (Tebé, Cabré \& Quiroz 2002), exponíamos cómo la necesidad de registrar los problemas terminológicos que se encuentran en una traducción había conducido a la elaboración de dos tipos de recursos, claramente diferenciados según su punto de partida: la constitución de glosarios del traductor, y la constitución de glosarios para el traductor.

En el primer caso, se trataba de glosarios que registraban las diversas soluciones propuestas por el traductor durante la elaboración de su traducción. Las características prototípicas del glosario del traductor son las siguientes: presenta un formato muy simplificado, casi siempre bilingüe; contiene equivalencias léxicas (términos, colocaciones, fraseología...), observaciones de uso, y a veces términos rechazables (errores de traducción, falsos amigos). La variación léxica que contiene está muy controlada o reducida a la mínima expresión. Los glosarios del traductor a menudo son almacenados en formato textual simple, en el mismo procesador de textos con que se ha resuelto la traducción (en forma de tablas o columnas), o en una hoja de cálculo, en formato tabulado. En síntesis, se trata de glosarios muy vinculados a cada traducción y/o a cada traductor, en el que las soluciones y equivalencias registradas tienen que ver sobre todo con la preocupación por resolver de manera coherente y armónica los problemas planteados en la traducción del texto, controlar interferencias léxicas o evitar posibles errores terminológicos.

Los glosarios para el traductor son glosarios mucho más sistemáticos, elaborados con metodología de trabajo terminológica, que contienen informaciones útiles para la toma de decisiones del traductor, en un formato monolingüe con equivalencias, bilingüe o plurilingüe. Estos glosarios, vocabularios, diccionarios... pueden contener muchas informaciones útiles para el traductor, como información contextual: contextos, 
colocaciones, notas de uso; información semántica: definiciones, conceptos relacionados, marcas temáticas; información gramatical: categoría gramatical, proceso de formación; e información pragmática: marcas de variación formal, profesional, geolectal, códigos de fiabilidad, etc. Las características de estos repertorios léxicos para la traducción son por lo tanto bien diferentes de los glosarios anteriores: formato más complejo, a menudo bajo una estructura de base de datos relacional; carácter bilingüe o plurilingüe; mucha más variación léxica, a menudo ponderada o contextualizada; informaciones que describen diferentes niveles de análisis de la unidad léxica; y almacenamiento en aplicaciones específicas de gestión de bases de datos.

El resultado de la elaboración de estos dos productos - el glosario del traductor y el glosario para el traductor - es la coexistencia de dos entornos de trabajo físicamente separados, las traducciones y los términos, que obligan a una navegación constante entre dos interfaces - el editor bilingüe y el gestor de bases de datos -, sin conexiones físicas entre ellas (de los textos a los términos, de los términos a los textos). En los glosarios para la traducción, además, se dedican esfuerzos importantes por reproducir los contextos de uso real de la terminología recogida en los textos, lo cual es un gasto de energía considerable. De hecho, como han señalado algunos autores (Srinivasan 1993: 393-394), el traductor necesita menos información fragmentada, tal y como aparece en muchos diccionarios y bases de datos, y más información contextualizada, en su propio entorno.

Esta separación física de los entornos de textos y términos hace que el traductor no dedique el tiempo necesario al mantenimiento y postedición de los datos terminológicos que son de su interés, por lo cual se pierde lo que vamos a denominar su memoria terminológica, que queda diluida, por un lado, entre múltiples segmentos de texto en varias lenguas, y por el otro, entradas de glosario sin ninguna relación ni estructuración interna entre ellas.

\section{La terminología en las memorias de traducción}

En nuestro primer trabajo en esta línea expusimos cómo están configurados los sistemas de traducción asistida basados en memorias de traducción, y qué posibilidades presentan para gestionar la terminología desde la traducción. Desde un punto de vista metodológico, defendíamos que los programas TAO eliminaban la concepción separada de los dos elementos (términos y textos), permitiendo una interacción de los textos a los términos y de los términos a los textos. La terminología se puede así recuperar y reutilizar de diferentes maneras para diferentes traducciones y conjunto de traducciones (agrupadas en proyectos de traducciones).

El resultado es que la constitución de glosarios del traductor resulta prácticamente innecesaria con la configuración que tenían antes, 
porque la mayoría de necesidades que justificaban la constitución de un glosario quedan satisfechas en el interior de la propia traducción. Así, los pares bilingües alineados permiten la consulta del término en su contexto, examinar diferentes colocaciones, o detectar unidades de traducción de dimensiones variables (unidades fraseológicas, oracionales...).

Por otro lado, las informaciones propias de los glosarios para el traductor se pueden presentar en forma de textos paralelos alineados, incorporando elementos de búsqueda e interrogación terminológica. La función básica de las informaciones contenidas en los glosarios/vocabularios/bancos de datos tradicionales era proporcionar muchas informaciones útiles para los traductores dentro un registro terminológico. Buena parte de estas informaciones, de carácter contextual y pragmático (colocaciones más habituales, selección de equivalentes, y otros) pueden inferirse directamente por el traductor desde un corpus de textos alineados que incorpore elementos de búsqueda e interrogación terminológica. Y cuando sea necesario, estas búsquedas se pueden efectuar directamente sobre Internet utilizando el inmenso corpus de validación de textos especializados disponibles en la red (cfr. Cabré et al. 2002) .

En síntesis, entendemos que un traductor necesita constituir un único tipo de glosario con el que, navegando entre términos y textos, pueda alimentar, recuperar y actualizar permanentemente la terminología utilizada en los textos que traduce.

\section{La elaboración de un glosario terminológico bilingüe desde la tra- ducción}

A continuación, analizamos de qué fases debería constar la elaboración de un glosario terminológico bilingüe elaborado con las herramientas de un sistema de traducción asistida basado en memoria de traducción. Nuestro punto de partida va a ser el siguiente: el traductor ya dispone de una pequeña memoria de traducción, es decir, parte de una serie de traducciones realizadas, alineadas por segmentos y procesadas por el sistema de traducción asistida. Igualmente, dispone de un pequeño glosario bilingüe que contiene algunas de las equivalencias registradas en esas traducciones.

\subsection{Fase de análisis y reconocimiento del texto}

El análisis previo del texto. Las herramientas de análisis textual que incorporan los entornos TAO permiten realizar un reconocimiento del texto contra las traducciones previamente resueltas por el mismo traductor. De esta manera, se obtienen estadísticas muy útiles, que indican el porcentaje de segmentos de texto que pueden recuperarse de la memoria, o el grado de repetición léxica que tiene el texto. Pero además, podemos saber: 
- los segmentos reconocidos en la memoria (es decir, idénticos al $100 \%$ )

- los segmentos parecidos que hay en la memoria (en un porcentaje de similaridad ajustable por el propio traductor)

- los segmentos no reconocidos en la memoria.

El análisis previo de la terminología. - Los paquetes TAO incorporan actualmente herramientas de reconocimiento terminológico, $\mathrm{y}$, en algunos casos, extractores rudimentarios de terminología. Tras aplicar estas herramientas, el traductor obtiene:

- una lista de unidades léxicas simples del texto, ordenadas por frecuencia

- una lista de las unidades terminológicas reconocidas en el glosario

- una lista de candidatos a término (en el caso del extractor).

El resultado conjunto de la aplicación de las herramientas de análisis y reconocimiento de textos y términos facilita al traductor una serie de elementos de análisis que le permitirá tomar decisiones importantes a la hora de abordar la traducción, como son:

- El grado de novedad del texto a traducir con respecto a la memoria (hay muchos, pocos, casi ningún segmento idéntico en la memoria...)

- El grado de novedad de la terminología del texto con respecto al glosario (hay muchos, pocos, casi ningún término registrado en la memoria...)

- La naturaleza de algunas de las unidades de traducción del texto, y su problemática de traducción - en algunos segmentos puede haber unidades de naturaleza muy variable, como unidades oracionales específicas, unidades adverbiales, colocaciones...

- El grado de repetición léxica de algunas unidades, y por lo tanto el peso de determinadas unidades en la traducción.

Así, el traductor puede visualizar con una cierta seguridad qué tipo de problemas deberá resolver, y qué tipo de recursos necesitará tener a mano, y en suma calcular qué esfuerzo le va a representar el trabajo de traducción y quizá, de forma orientativa, en qué tareas se repartirá este trabajo.

Pero además, el traductor puede sin duda afinar los criterios de selección de las unidades que potencialmente son candidatas a ir identificadas y etiquetadas en el glosario: las más frecuentes en el texto, las que son semánticamente más opacas antes de la traducción, las que pueden presentar polisemia, las que pueden presentar variación geolectal, etcétera. 


\subsection{Fase de pretraducción}

En la fase de pretraducción, el traductor interviene sobre los parámetros de la memoria para activar la sustitución de los segmentos del texto a traducir. Esta fase no conlleva ninguna intervención cualitativa importante por parte del traductor, puesto que es la máquina la que realiza la sustitución de forma autónoma; sin embargo sus consecuencias pueden ser importantes, en la medida en que la traducción automática de todos los segmentos por encima de un determinado umbral de similaridad significa que posteriormente el traductor no revisará esos segmentos - o, aunque lo haga, es posible que no detecte muchos problemas de traducción o de terminología que sí hubiera detectado al replantearse la traducción de esos segmentos.

En concreto, en la fase de pretraducción, el traductor puede

- traducir automáticamente los segmentos que presenten un $100 \%$ de similaridad con la memoria - y marcarlos como ya traducidos.

- traducir parcialmente aquellos segmentos que presenten un alto grado de similaridad - y marcarlos como pretraducidos o traducidos parcialmente, pendientes de verificación.

- sustituir, dentro de cada segmento, las unidades terminológicas del texto a traducir que presenten una equivalencia en la llengua de llegada recopilada en el glosario ya constituido.

Esta última decisión es muy aconsejable desde nuestro punto de vista, puesto que permitirá trabajar al traductor aplicando la prueba de la sustituibilidad de los términos de la lengua origen por las unidades terminológicas del glosario, lo que le permitirá verificar al momento su validez para la traducción en curso.

\subsection{Fase de traducción}

En la fase de traducción propiamente dicha, el traductor, situado en la ventana correspondiente a la lengua meta, y con los demás elementos del entorno de traducción convenientemente activados - gestor terminológico, bloc de notas, acceso a Internet - empieza a recorrer los segmentos de la lengua origen validando pretraducciones, completando traducciones parciales o traduciendo de nuevo, según sea el caso.

En esta fase es cuando se produce una interacción mayor entre la memoria de traducción - que alimenta con propuestas cada nuevo segmento que hay que traducir - y la memoria terminológica - que alimenta desde el glosario propuestas de equivalencia que coincidan con un segmento entero o, más frecuentemente, con una unidad que se halle dentro des sus límites. 
En la fase de traducción, la interacción que describimos es ahora bidireccional: por un lado, como se ha visto, el glosario proporciona propuestas de equivalencia para el texto que se está traduciendo. Por el otro, el traductor puede decidir incorporar al glosario durante la traducción las equivalencias que considere pertinentes desde el texto hacia el gestor de base de datos. La primera interacción, como se ha dicho, se dirige desde la memoria de traducción y el glosario hacia el texto, de modo que su resultado no tiene influencia directa sobre el glosario. Pero la segunda interacción posible se dirige hacia el glosario, puesto que el traductor puede ir introduciendo equivalencias desde el editor bilingüe de la traducción hacia el gestor de base de datos. Si el traductor tiene interés en mantener una memoria terminológica a la par que una memoria de traducción, es imprescindible que prevea la necesidad de introducir esos datos durante el proceso de traducción, puesto que después, buena parte de las equivalencias terminológicas que habrá resuelto traduciendo quedarán escondidas dentro de los segmentos en cuestión, y no podría recuperarlas en una nueva traducción salvo que se encontrara con un segmento idéntico al de la memoria, o por lo menos con un coeficiente de similaridad muy alto al que tiene en la memoria.

La motivación para introducir una equivalencia terminológica en el glosario puede responder a distintas necesidades o criterios, de acuerdo con los intereses del traductor. Los casos más frecuentes son:

- la unidad terminológica en la lengua origen tiene una alta frecuencia de aparición en el texto (detectado en la fase de análisis), y resulta económico aislarla en el glosario para que aparezca inmediatamente como propuesta en los sucesivos segmentos del mismo texto.

- la unidad terminológica presenta una equivalencia en la lengua meta que ha sido difícil resolver (o que es una propuesta del traductor, distinta de la que está registrada en otras fuentes), y resulta conveniente aislarla y registrarla.

- la unidad terminológica presenta distintas equivalencias posibles en la lengua meta (por razones de tipo geolectal, por ejemplo), y resulta conveniente registrar todas sus posibilidades a fin de que el traductor visualice en cada momento de las posibilidades que tiene a su alcance -evitando errores de traducción, o empobreciendo su traducción con una misma equivalencia en la lengua meta una y otra vez.

- la unidad terminológica presenta en la lengua meta una equivalencia errónea bajo la forma de un falso amigo, un error de traducción en el que el traductor advierte que puede caer fácilmente, de modo que resulta conveniente que el glosario recuerde al traductor la equivalencia correcta cada vez que esa unidad aparezca de nuevo en la lengua origen.

La creación o alimentación de un glosario desde el proceso de traducción puede hacerse de diferentes maneras, según los distintos programas de 
traducción asistida que hay en el mercado, pero básicamente hay dos posibilidades:

Entrada de datos directa. - El proceso de entrada de datos se puede hacer de manera rápida con las propias utilidades del programa marcando las unidades terminológicas en el texto de partida y en el texto de llegada. Este proceso de entrada rápida permite crear un glosario de forma inmediata, con unos contenidos mínimos, que podrá ser revisado posteriormente. Las últimas versiones incorporan un extractor para inventariar la terminología de la traducción en forma de glosarios paralelos con candidatos a términos, importarla a la base de datos, editarla si es conveniente y reutilizarla posteriormente.

Entrada de datos indirecta. - El proceso de entrada de datos se puede hacer con una interfaz específica, más compleja, externa al programa que se está utilizando. Este módulo adicional facilita la introducción de distintos campos de forma guiada, e interacciona entre el editor bilingüe de la traducción y el glosario.

\subsection{Fase de postedición}

Una vez la traducción está terminada, el traductor ha generado dos productos que deben ser revisados cuidadosamente: la traducción en sí misma (para la que dispone de un conjunto de herramientas de revisión) y el glosario. Recordemos que el glosario creado o alimentado durante el proceso de revisión está necesariamente incompleto, y deben realizarse distintas tareas de revisión. Esta fase del trabajo es crítica, puesto que la configuración de la memoria terminológica depende básicamente. Enumeramos las más importantes:

Verificación de la consistencia terminológica entre texto y glosario. - Este aspecto de la revisión pretende controlar que las equivalencias terminológicas utilizadas en contexto, es decir, en el interior de cada par bilingüe de segmentos, coincida con las equivalencias que recoge el glosario. La situación más habitual es que no siempre coincidan al $100 \%$ por las razones expuestas más arriba: es perfectamente posible, por ejemplo, que el traductor haya ignorado una propuesta de equivalencia terminológica procedente del glosario porque no le parecía adecuada, y que al resolverla según su criterio dentro del segmento que estaba traduciendo no la hubiera incorporado al glosario. En este caso, la memoria terminológica queda afectada, ya que la inconsistencia puede afectar a la futura coherencia terminológica de las nuevas traducciones. Para ello, algunos programas disponen de funciones de verificación de la consistencia terminológica, que permiten revisar todo el texto traducido comprobando en cada caso si las equivalencias del texto meta y las propuestas del glosario coinciden.

Compleción y revisión del glosario. - Para que el glosario creado o alimentado durante la traducción pueda ser reutilizado con garantías 
debe enriquecerse con informaciones - de carácter cualitativo, sobre todo - que faciliten la toma de decisiones posteriores sobre el uso de las equivalencias que contiene. En nuestra opinión, son más necesarias observaciones sobre las condiciones de uso de las variantes recogidas, o una ponderación de las distintas opciones de traducción según el estatus del término antes que añadir definiciones (que pueden obtenerse fácilmente en Internet) o contextos (la propia memoria de traducción ya permite la consulta de colocaciones, es decir, del término en su contexto inmediato de uso).

Especificación de su ámbito de aplicación. - Un aspecto muy importante que permiten los programas TAO, es etiquetar los pares de segmentos del texto traducidos según una serie de parámetros que permiten posteriormente su filtrado y recuperación selectiva. Por ejemplo, una traducción en un SMT puede ser etiquetada por el cliente que la ha encargado, por la temática de que trata, o por el grado de formalidad del texto, entre otros criterios.

Así, un etiquetado cuidadoso de las traducciones bajo estos u otros parámetros complementarios permite que uno vaya configurando y dimensionando la memoria de traducción que desea utilizar en cada momento, incluyendo o excluyendo partes de la memoria según se ajusten más o menos a los requerimientos del nuevo proyecto de traducción.

En nuestra opinión, para convertir uno o distintos glosarios del traductor en una verdadera memoria terminológica sería necesario que las unidades terminológicas del o de los glosarios pudieran etiquetarse igualmente según distintos parámetros, de modo que para cada necesidad de traducción el traductor dispusiera de una selección de la terminología más adecuada para tal fin.

Subrayamos que estas herramientas no existen en este momento, y cualquier operación de, por ejemplo, subdivisión de un glosario en dos o más glosarios a partir de cualquier criterio mencionado anteriormente debería hacerse de forma manual, etiquetando cada unidad y luego procediendo a la exportación de las unidades, o su fusión con otros glosarios.

Pero esta opción permitiría, por ejemplo, recuperar de una memoria terminológica constituida sobre textos de informática:

- a terminología bilingüe inglés-español normalizada de los microprocesadores

- las colocaciones verbales inglés-portugués de los manuales de instrucciones de software

- la terminología que utiliza Microsoft -versus la terminología que utiliza Apple

- toda la terminología microinformática utilizada en la década 19902000

- todos los homónimos del ámbito de la digitalización de imágenes 


\section{Conclusiones, perspectivas}

En esta comunicación hemos querido presentar de qué modo las nuevas herramientas de traducción especializada cambian la gestión de la terminología elaborada por el traductor, y para finalidades de traducción. Hasta hace poco tiempo, la elaboración de terminología en la traducción adolecía de muchos defectos: equivalencias ad-hoc, criterios de selección erráticos, falta de consistencia y sistematicidad de los datos, formato rígido, nula integración con la traducción, dificultades insalvables en tiempo y esfuerzo para reutilizar dicho material en otras traducciones. Y por otro lado, la terminología para la traducción, elaborada bajo una metodología sistemática rigurosa, propia de la disciplina, presentaba dificultades para servir, en su concepción actual, a los propósitos y necesidades de la traducción: criterios de selección de unidades ajenos a los problemas de traducción, énfasis en los aspectos de codificación semántica - definiciones, áreas temáticas, notas de uso - y escasa información contextualizada que aporte información pragmática sobre el comportamiento de esas unidades para un traductor.

La metodología de elaboración de terminologías bilingües en programas de memoria de traducción, de la que hemos presentado aquí un primer esbozo, nos proporciona la posibilidad de crear un único recurso que integre de forma dinámica la consulta sobre unidades terminológicas y unidades textuales, sobre las bases siguientes:

- criterios de selección ajustados a las necesidades del texto a traducir: unidades terminológicas más frecuentes, unidades muy poco frecuentes pero pertinentes en el texto, unidades terminológicas que presentan equivalencias problemáticas, candidatos a término procedentes de las herramientas de extracción...

- alimentación del glosario directamente procedente de la traducción: las equivalencias resueltas por el traductor pueden introducirse directamente en cualquiera de los glosarios activos, bien constituyendo un nuevo registro, bien enriqueciendo (o corrigiendo) una entrada existente con una nueva equivalencia. Y todo ello, sin salir del propio entorno de traducción

- verificación inmediata de la validez de las propuestas terminológicas: cuando el glosario actúa como fuente de propuestas para las unidades terminológicas de un segmento que se va a traducir, la sustitución de todas las unidades terminológicas de la lengua origen por sus equivalencias en la lengua meta contenidas en el glosario permite que el traductor haga un ejercicio constante de validación de las propuestas registradas en el gestor; si detecta que no son válidas y debe cambiarlas, puede registrar las nuevas propuestas en el mismo momento en que las resuelve

- comprobación de la consistencia entre el texto y los términos: pueden controlarse los posibles desajustes entre la terminología uti- 
lizada en el texto y la terminología registrada en el glosario, y que es normal que se produzca durante la resolución de la traducción, de modo que no serán necesarias costosas revisiones posteriores para asegurar la adecuación de la terminología al texto

- posibilidades de postedición abiertas: después de la resolución de la traducción, los gestores de terminología utilizados en la traducción ofrecen exactamente las mismas posibilidades de revisión que los gestores terminológicos autónomos - es más, en algunos casos son la misma herramienta.

En futuros trabajos en esta misma línea nos proponemos definir más profundamente las consecuencias metodológicas que puede tener la constitución de una memoria terminológica, así como los nuevos interrogantes que plantearía su implantación tanto en entornos profesionales como en la docencia de la terminología en entornos de traducción especializada.

\section{Bibliografía}

Cabré, M.T. (1999). La terminología: representación y comunicación. Una teoría de base comunicativa y otros artículos. Barcelona: IULA Universitat Pompeu Fabra (Sèrie monografies, 3).

Cabré, M.T., R. Estopà, J. Freixa,, M. Lorente \& C. Tebé (2002). "Les necessitats terminològiques del traductor científic". Translating Science. Proceedings: 2nd International Conference on Specialized Translation Barcelona: PPU

Sager, J. C. (1990). A Practical Course in Terminology Processing. Amsterdam / Philadelphia: John Benjamins.

Srinivassan, S. (1993). Developing Terminology Management Software for Translators. In: K.-D. Schmitz (ed.) TKE' 93. Indeks Verlag

Tebé, C. (2003). "Las herramientas informáticas en didáctica de la traducción especializada". M. Á. García Peinado \& E. Ortega Arjonilla (eds.) Panorama actual de la investigación en traducción e interpretación. (Volumen II). Granada: Editorial Atrio, S.L

Tebé, C., M.T. Cabré \& G. Quiroz (2002). "La gestió de la terminologia en les memòries de traducció". Translating Science. Proceedings: 2nd International Conference on Specialized Translation. Barcelona: PPU 
